\title{
Evaluation of the Impact That PARs Have on the Hydrogen Risk in the Reactor Containment: Methodology and Application to PSA Level 2
}

\begin{abstract}
Ahmed Bentaib, Cataldo Caroli, Bernard Chaumont, and Karine Chevalier-Jabet
Service d'évaluation des Accidents Graves et des rejets Radioactifs, Institut de Radioprotection et de Sûreté Nucléaire, Direction de la Sûreté des Réacteurs, BP 17, 92262 Fontenay aux Roses, France

Correspondence should be addressed to Ahmed Bentaib, ahmed.bentaib@irsn.fr

Received 16 April 2009; Revised 8 February 2010; Accepted 11 May 2010

Academic Editor: Michel Giot

Copyright ( $\odot 2010$ Ahmed Bentaib et al. This is an open access article distributed under the Creative Commons Attribution License, which permits unrestricted use, distribution, and reproduction in any medium, provided the original work is properly cited.

This paper presents a methodology and its application to a Level 2 Probabilistic Safety Assessment (PSA-2), to evaluate the impact of the Passive Autocatalytic Recombiners (PARs) on the hydrogen risk in the reactor containment in case of a severe accident. Among the whole set of accidental scenarios calculated in the framework of the PSA-2, nine have been selected as representative in terms of the in-vessel hydrogen production rate and in-vessel total produced hydrogen mass. Five complementary scenarios have been added as representative of the core reflooding situations. For this set of selected scenarios the evolution of the conditions in the containment (i.e., pressure, temperature, and composition) during the in-vessel phase of the accident has been evaluated by means of a lumped parameter approach. The use of spray systems in the containment has also been considered as well as the presence of recombiners. Moreover, the ignition by recombiners of the flammable atmosphere has been considered.
\end{abstract}

\section{Introduction}

In the theoretical case of a severe accident in a nuclear reactor with core meltdown, the interaction of the hot core with the cooling water can generate large amounts of hydrogen. Hydrogen may be produced by oxidation of metals present in the corium pool or in the base mat during the molten corium-concrete interaction phase. This hydrogen is transferred into the containment (and transported therein) by convection loops arising mainly from condensation of steam released via the RCS break or during corium-concrete interaction. Depending on mixing in the containment atmosphere, the distribution of hydrogen is more or less homogeneous. If considerable hydrogen stratification exists, local concentrations of hydrogen may become substantial, exceeding the lower flammability limit for the gas mixture. The distribution and concentration of hydrogen in the containment building may also be modified by its spray systems. Spraying does homogenize the distribution of hydrogen in the containment and lead to "deinertization" of the mixture through the condensation of steam on water droplets.

To limit the hydrogen concentration in the containment, several methods can be proposed [1]:

(i) the deliberate ignition of the mixture as soon as the flammability limit is reached,

(ii) the consumption of hydrogen,

(iii) the removal of oxygen,

(iv) the dilution of the atmosphere to prevent the formation of flammable mixtures either by the increase in the volume of the containment or by the injection of an inert gas.

Hydrogen risk management can be implemented by one or a combination of the previous methods. The choice of a mitigation strategy depends primarily on the design of the containment. For PWRs with large dry containment, the strategy usually consists in combining large free volume to allow dilution, a high value of the design pressure, and the 
use of means, as passive autocatalytic recombiners (PARs), to consume hydrogen. This strategy has been adopted recently in all French PWRs.

In this paper, we propose a methodology to evaluate the impact of PARs on the hydrogen risk in French 900 MWe reactor containment in case of a severe accident and its application to a Level 2 Probabilistic Safety Assessment (PSA-2).

\section{Hydrogen Risk Assessment Methodology}

The methodology adopted to assess hydrogen risk in the reactor building must take into account the different loads accounting for the impact of hydrogen production, distribution, and mitigation systems. This method uses the following main steps [2].

Step 1. Plant Design. The starting point of any analysis is the selection of the plant and geometrical modeling of the containment. This step aims to well describe the containment shape and volume; which influence hydrogen distribution inside the reactor containment.

Step 2. Selection of Relevant Scenarios. Representative of severe accident sequences and the evaluation of the associated hydrogen production rates and release into the reactor building. These source terms are usually derived from parametric code calculations with best estimates for still uncertain hydrogen production processes.

Step 3. Evaluation of the Containment Atmosphere Conditions. During the accident transient (i.e., temperature, pressure, and gas composition in the different regions and volumes of the containments) accounting for the presence of mitigation systems.

Step 4. Evaluation of the Time Evolution of Flammable Hydrogen-Air-Steam Cloud. The flammability of the containment gas mixture depends on its temperature, pressure, and composition. However, in practice, the point representing the mixture's composition (hydrogen, air, and steam) on the Shapiro diagram [3] (see Figure 1) is used to determine whether the mixture is flammable. In this diagram, the flammability and detonation zones are, respectively, delimited by the exterior and interior curves.

Step 5. Evaluation of the Propensity of a Premixed Flame to Propagate inside the Containment. Under the effect of hydrodynamic instabilities and turbulence (caused primarily by obstacles in the flame's path), an initially laminar deflagration (with a flame velocity around $1 \mathrm{~m} / \mathrm{s}$ ) may accelerate. Fast combustion regimes may also develop, involving rapid deflagration (a few hundred $\mathrm{m} / \mathrm{s}$ ), deflagration-to-detonation transition (DDT) and detonation (over $1000 \mathrm{~m} / \mathrm{s}$ ). These combustion regimes may generate high pressure loads which could endanger the containment integrity.

To define the transition from slow to fast combustion regime, two types of criteria, based on numerous experiments, are considered $[2,4]$.

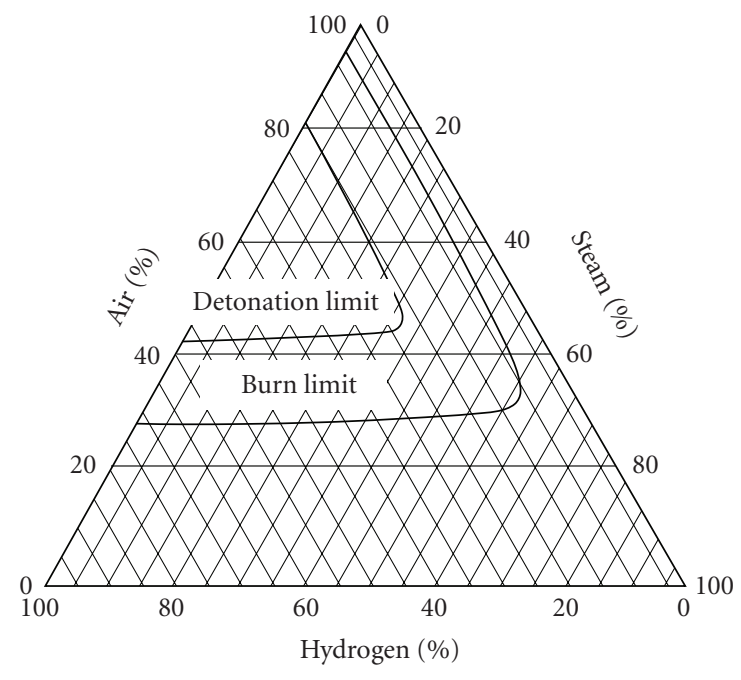

FIGURE 1: Shapiro diagram for hydrogen-air-steam mixtures.

(i) The " $\sigma$ " criterion related to flame acceleration. $\sigma$ stands for the mixture's expansion factor, a ratio of fresh and burnt gas densities at constant pressure. It is an intrinsic property of the mixture.

The critical value $\sigma^{*}$ beyond which flame acceleration is possible depends on initial gas composition and temperature and flame stability (see Figure 2).

(ii) Similarly, prerequisite conditions have been defined for characterizing the transition between deflagration and detonation regimes (DDT). They are based on comparing a characteristic dimension of the geometry with detonation cell size $\lambda$ (see Figure 3 ).

Flame acceleration and DDT criteria are based on the results of numerous experiments at various scales and in various geometries [5] and are considered as prerequisite criteria, that is, conditions required for the various combustion modes.

Step 6. Evaluation of Pressure and Thermal Loads Generated by Combustion. Two configurations are distinguished.

(i) If flame acceleration criteria are not met: in this case, dynamic pressure loads are excluded, and the pressure load is evaluated by considering adiabatic complete isochoric combustion process.

(ii) If flame acceleration criteria are met: in this case, the induced combustion loads are evaluated using the most appropriate combustion models.

\section{Impact of PARs on Hydrogen Risk}

In the following, the previous methodology is applied to investigate the PARs impact on hydrogen risk in French 900 MWe reactor containment by considering severe accidents sequences simulated in frame of PSA level 2. Effect of spray actuation and core reflooding is also considered. 


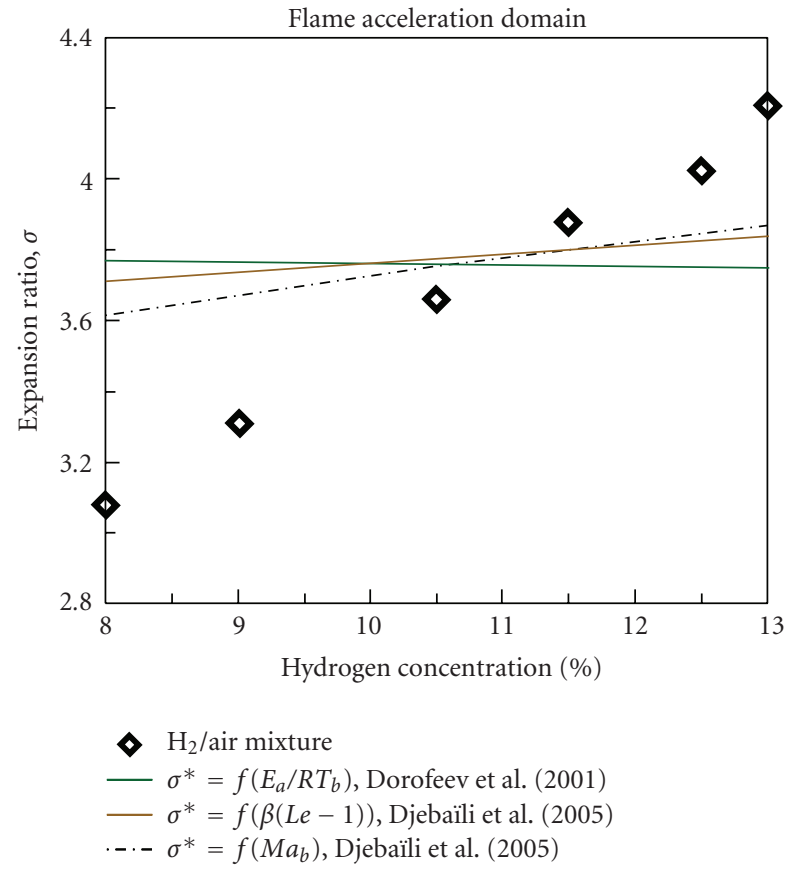

Figure 2: Critical value $\sigma^{*}$ as a function of hydrogen concentration.

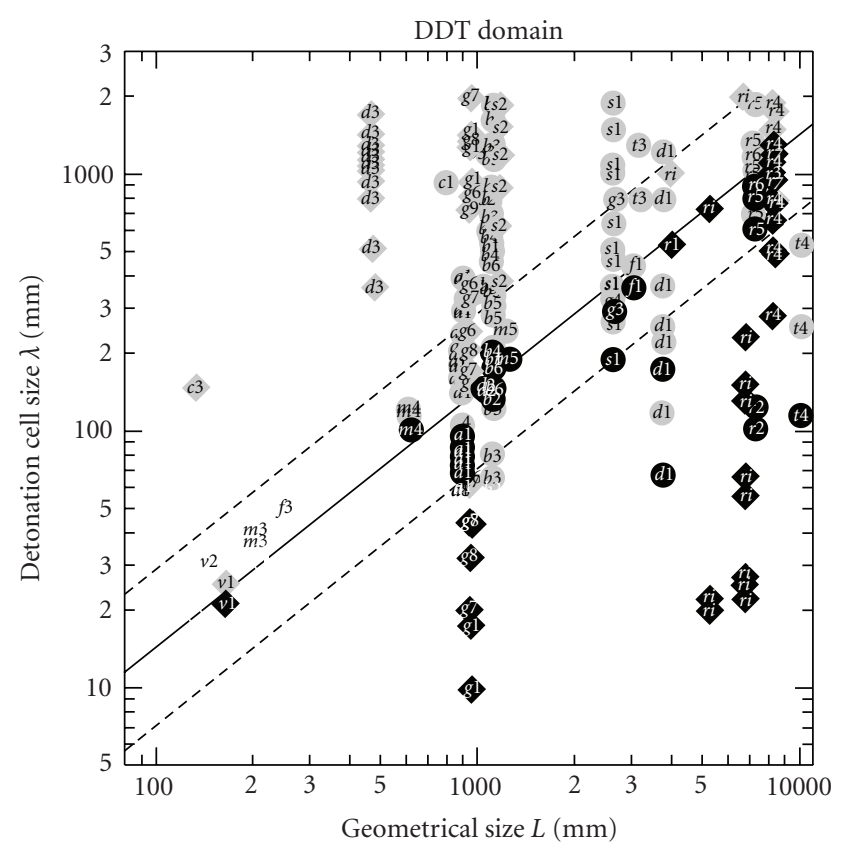

No DDT, BR $<0.5$

DDT, BR $>0.5$ and rooms

No DDT, BR $>0.5$ and rooms $-L=7 \lambda$

DDT, $B R<0.5$

$---\lambda$ accuracy limits

FIgURe 3: DDT criteria.

All calculations are performed using ASTEC V1.1 Lumped parameter code jointly developed by IRSN and GRS, that is, Gesellschaft für Anlagen- und Reaktorsicherheit mbH and dedicated to the simulation of the whole course of severe accidents in light-water reactors [6].
3.1. Step 1. Plant Design. The reactor containment is modeled by using multicompartment approach (see Figure 4). This model is based on 65 elementary volumes by considering 6 vertical levels, various radials sections, and 3 azimutal sections corresponding to the 3 reactor loops [7].

Moreover, the structure of the casemates surrounding each of the 3 primary loops is described. In addition to elementary volumes and in order to take into account atmosphere wall heat and mass transfer, 450 concrete and steel walls were considered. For gas and liquid transports inside the containment, 270 junctions connecting zones were modeled.

3.2. Step 2. Scenarios Definition. From the analysis of PSA level 1 results, 35 scenarios had been identified as sequences leading to core degradation and hydrogen release inside the reactor containment. Theses core degradation sequences correspond to the following situations:

(i) accidents involving secondary circuit transients,

(ii) accidents involving loss of steam generators feedwater,

(iii) accidents involving steam generator tube ruptures SGTR,

(iv) accidents involving loss of coolant LOCA,

(v) accidents involving loss of electrical power.

Theses sequences lead to different amount of hydrogen with different kinetic of hydrogen release (see Figure 5).

As PARs efficiency is related to the atmosphere composition and thermal hydraulic conditions [8], the analysis of PARs impact on hydrogen risk should take into account several parameters as (pressure, steam, and oxygen concentrations). Nevertheless, the main important effect remains the hydrogen total mass and the kinetic of hydrogen release inside the containment. To take into account those parameters, classification according to hydrogen production rate and hydrogen mass is carried out. The adopted approach distinguishes three levels of hydrogen mass:

(i) Weak corresponding to a mass of hydrogen produced lower than $300 \mathrm{~kg}$,

(ii) Medium corresponds to a produced hydrogen mass between $300 \mathrm{~kg}$ and $550 \mathrm{~kg}$,

(iii) High corresponds to a mass of hydrogen produced higher than $550 \mathrm{~kg}$,

and three levels of hydrogen production rate:

(i) Weak for $\mathrm{H} 2$ production rate lower than $0.1 \mathrm{~kg} / \mathrm{s}$,

(ii) Medium for $\mathrm{H} 2$ production rate between $0.1 \mathrm{~kg} / \mathrm{s}$ and $0.15 \mathrm{~kg} / \mathrm{s}$,

(iii) High for $\mathrm{H} 2$ production rate higher than $0.15 \mathrm{~kg} / \mathrm{s}$.

The combination of the previous criteria leads to 9 types of scenarios as shown in Table 1.

The application of the previous criteria to the various scenarios allowed defining 9 families of accidental scenarios 


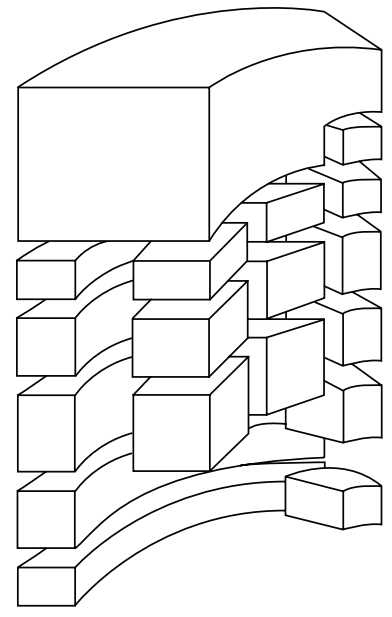

(a)

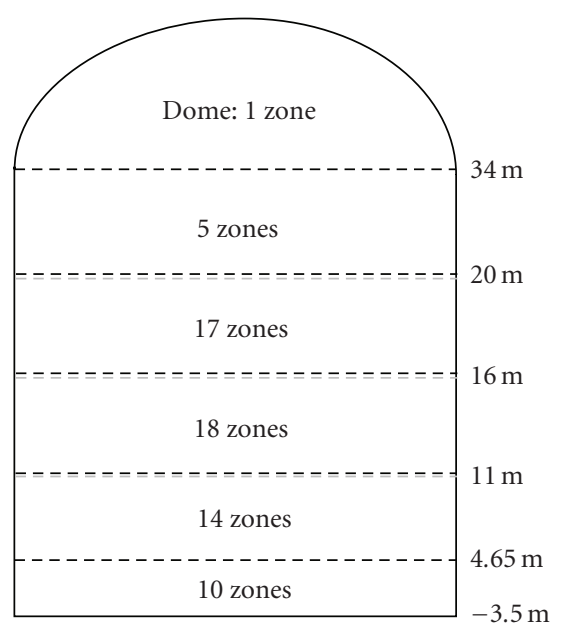

(b)

Figure 4: View of PWR 900 Astec nodalisation.

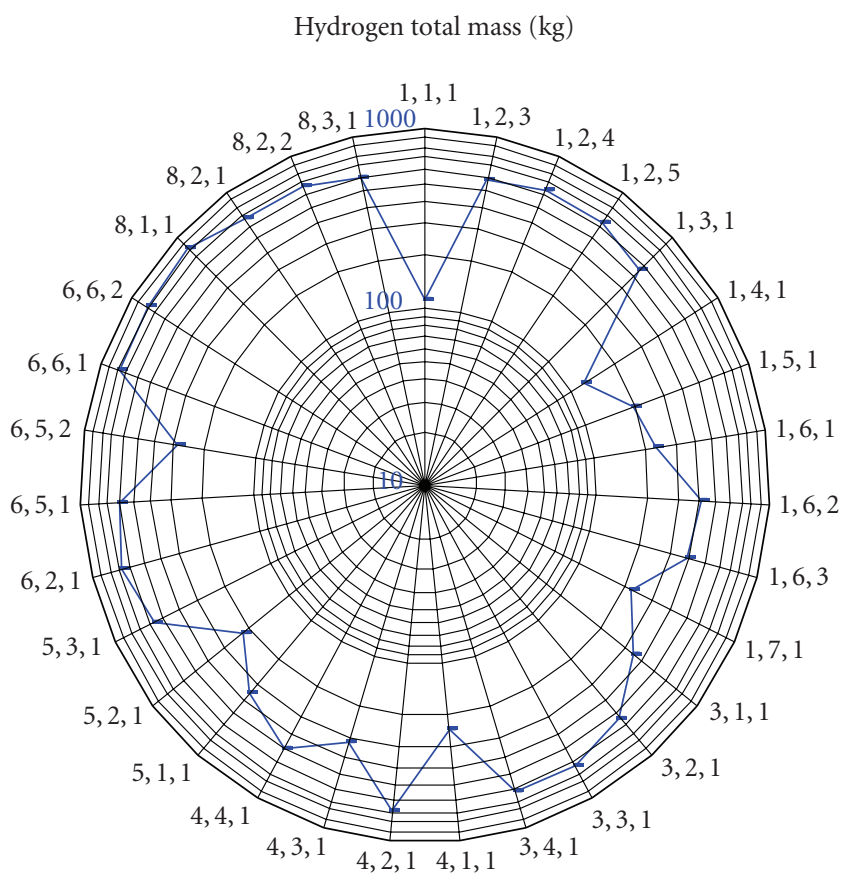

(a)

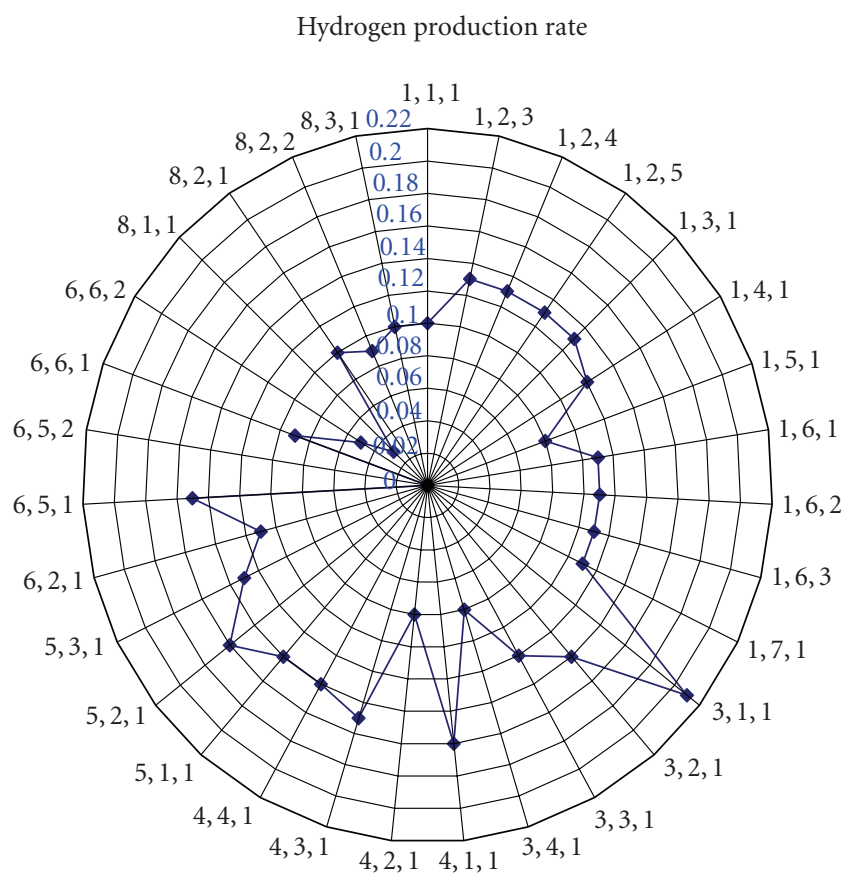

(b)

FIGURE 5: Hydrogen mass and hydrogen production rate for each core degradation sequences.

whose members present the same "behavior" regarding to hydrogen risk. Thus the PARs effect investigation will be limited to these 9 sequences.

In addition and to take into account core reflooding situations, 3 safety injection rates corresponding to $5.5 \mathrm{~kg} / \mathrm{s}$, $41.4 \mathrm{~kg} / \mathrm{s}$, and $205.6 \mathrm{~kg} / \mathrm{s}$ are considered. Moreover, spray actuation is considered as soon as gas pressure exceeds 2.4 bars.

3.3. Steps 3 and 4. Time Evolution of Flammable Clouds inside the Containment. The flammable gas time evolution is checked by considering gas mixture in each containment zone at each time in the ternary H2-Air-Steam diagram [3]. At each time, gas composition is represented by a point in the Shapiro diagram. The gas composition time evolution in each zone is then described by curve. Figure 6 shows the gas composition in a zone during the core degradation sequence by considering or not the use of PARs. This figure shows also that the use of PARs decreases the hydrogen concentration in the containment atmosphere and limits the flammable cloud size inside the reactor containment. 
TABLE 1: Families of scenarios.

\begin{tabular}{lcc}
\hline Families of scenarios & H2 mass & H2 production rate \\
\hline Type 1 & Weak & Weak \\
Type 2 & Weak & medium \\
Type 3 & Weak & High \\
Type 4 & Medium & Weak \\
Type 5 & Medium & medium \\
Type 6 & Medium & High \\
Type 7 & High & Weak \\
Type 8 & High & medium \\
Type 9 & High & High \\
\hline
\end{tabular}

More generally, the analysis of time evolution of reactor containment atmosphere regarding to PARs effect allows defining three classes of scenarios.

(i) Class 1: constituted of scenarios corresponding to weak productions of hydrogen and to reflooding scenarios with high safety injection rate $(205,6 \mathrm{~kg} / \mathrm{s})$. For this category, the use of PARs seems to be sufficient to avoid flammable gas formation.

(ii) Class 2: constituted of scenarios corresponding to medium and high productions rate. In these cases, flammable gas mixture is present in certain containment zone during a limited time.

(iii) Class 3: corresponds to reflooding scenarios with safety injection rate of $41.4 \mathrm{~kg} / \mathrm{s}$ and $5.5 \mathrm{~kg} / \mathrm{s}$. In these cases, containment gas atmosphere becomes flammable during reflooding phase.

3.4. Step 5. Flame Acceleration. As for the flammability risk assessment, flame acceleration risk is checked by calculating and comparing the expansion $\sigma$ factor, corresponding to the gas composition in each zone, to the limit value $\sigma^{*}$, issued from experimental data [4]: situation with $\sigma$ higher than $\sigma^{*}$ could lead to high pressure load in case of combustion.

The analysis of ASTEC results show that the use of PARs leads to low hydrogen concentration and consequently to low $\sigma$ values. This situation is illustrated in Figure 7 where $\sigma$ values obtained with and without PARs are compared to the critical values $\sigma^{*}$ at time of high hydrogen release for each zone.

As shown in Figure 7, the use of PARs allows to reduce significantly the flame acceleration risk. Nevertheless, the analysis of PARs effect shows that for

(i) class 1, the use of PARs avoids flame acceleration;

(ii) class 2, flame acceleration criterion is satisfied in certain containment zone during a limited time;

(iii) class 3, flame acceleration criterion is satisfied by the reactor containment gas atmosphere during reflooding phase.

Consequently, scenarios from class 1 could not lead to high pressure load in case of combustion. Indeed, scenarios from classes 2 and 3 may endanger the containment integrity

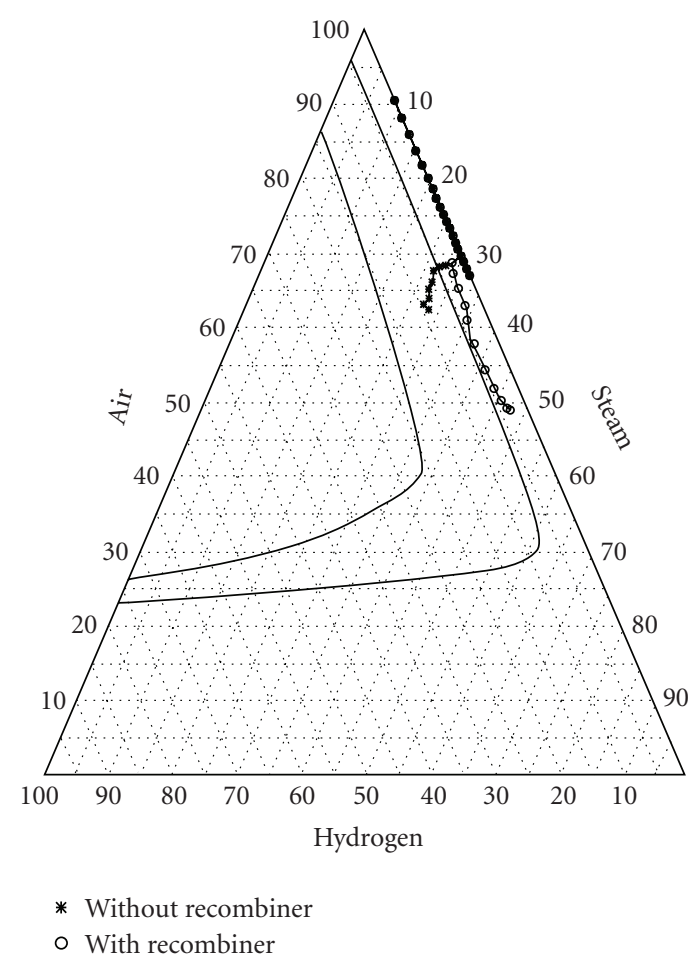

FIGURE 6: Effect of PARs on gas mixture flammability (with stars: gas composition without PARs; with circle: gas composition with PARs).

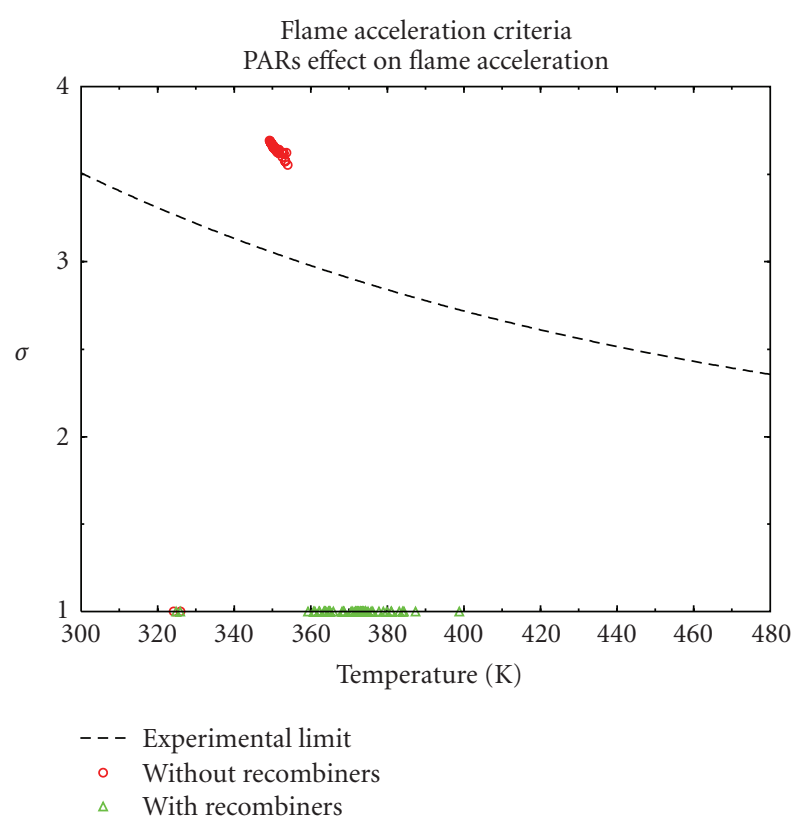

Figure 7: PARs effect on Flame acceleration (in red: gas composition without PARs; in green: gas composition with PARs).

in case of combustion if ignition occurs at moment of high hydrogen release.

3.5. Step 6. Pressure Loads Evaluation. Before performing combustion calculation, ignition sources have to be defined. 


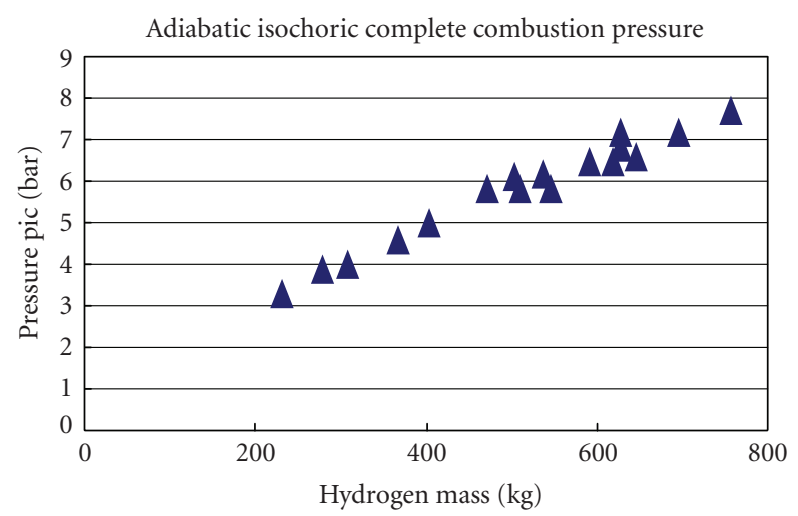

Figure 8: PAICC pressure versus hydrogen mass.

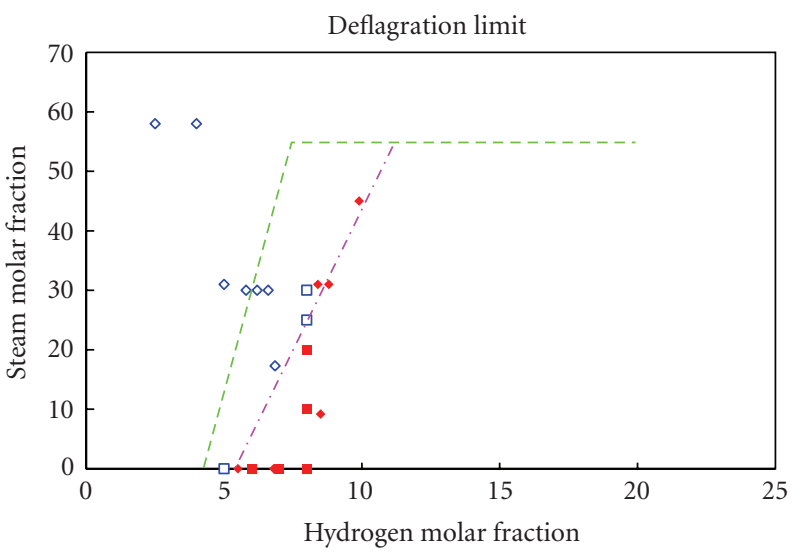

PARs ignition domain

$\diamond$ H2PAR-no deflagration - KALIH2-deflagration

$\square$ KALIH2 - no deflagration - - - Simplified flammability limit

- H2PAR—deflagration _... Deflagration limit

Figure 9: Ignition of SIEMENS PARs.

The ignition must be either predicted mechanistically (selfignition) or must be postulated with respect to time and location. In this last case, ignition time is usually chosen to induce high pressure load. Figure 8 shows the pressure maximal values obtained by considering adiabatic isochoric complete combustion for different core degradation sequences.

Figure 8 shows also that the "probabilistic" ignition sources could lead to high pressure values beyond the containment pressure design of 6.5 bars. In the framework of PSA level 2 and in order to evaluate "realistic" pressure load, deterministic ignition sources are considered. Due to their hot catalytic sheets, PARs are considered as ignition sources under specific condition (see Figure 9). Indeed, some of the experimental tests performed on KALI H2 and H2PAR [9] show that PARs could ignite the flammable gas mixture. These experimental results show that ignition induced by recombiners occurs for low hydrogen concentrations respecting to the following limits.

These ignition limits correspond to low hydrogen concentration which avoid flame acceleration phenomena. The evaluation of pressure load generated by combustion is then carried out by considering complete, adiabatic and isochoric combustion. Pressure, known as PAICC, thus calculated depends on gas mixture and on the thermodynamic conditions and does not depend on the geometry of the containment. The calculation results show that PAICC does not exceed the containment pressure design.

\section{Evaluation of Spraying Effect}

Spray system has been activated for the previously selected scenarios. The effect of this activation has been analyzed and shows that, for all scenarios, steam condensation on spray droplets leads to the creation of consequently flammable cloud. Moreover, flame acceleration criteria are satisfied in the whole of the reactor containment. In all cases, scenarios from classes 1 and 2 families behave as scenarios from class 3.

\section{Conclusion}

This paper presents a methodology for hydrogen risk assessment and its application to evaluate the effect of Passive Autocatalytic Recombiners in framework of Level 2 PSA.

This analysis has shown that the use of recombiners reduces significantly the risk of flame acceleration and transition to detonation. However, the presence of recombiners does not eliminate the risk of flame acceleration which lasts for some specific scenarios and short duration.

These analyses have also shown the beneficial effect of recombiners as igniters. Indeed, based on the experimental data presently available, it seems that ignition induced by recombiners occurs for low hydrogen concentrations, leading to relatively low pressure. These experimental results need however to be corroborated by more detailed experiments and by refined modelling of phenomena occurring in PARs.

\section{References}

[1] Research and development with regard to severe accidents in pressurized water reactors: summary and outlook, Tech. Rep. IRSN/2007-083.

[2] P. Royl, H. Rochholz, W. Breitung, J. R. Travis, and G. Necker, "Analysis of steam and hydrogen distributions with PAR mitigation in NPP containments," Nuclear Engineering and Design, vol. 202, no. 2-3, pp. 231-248, 2000.

[3] H. F. Coward and G. W. Jones, Limits of Flammability of Gases and Vapors, Bulletin 503, Bureau of Mines, United States Government Printing Office, Washington, DC, USA, 1952.

[4] N. Chaumeix-Djeibli, et al., H2 Gradient Effect on Premixed Flame Propagation in a Vertical Facility: ENACCEF ; ICDERS 20 Montréal 2005.

[5] W. Breitung, S. Dorofeev, A. Kotchourko, et al., "Integral large scale experiments on hydrogen combustion for severe accident code validation-HYCOM," Nuclear Engineering and Design, vol. 235, no. 2-4, pp. 253-270, 2005.

[6] J.-P. Van Dorsselaere, C. Seropian, P. Chatelard et al., "The ASTEC integral code for severe accident simulation," Nuclear Technology, vol. 165, no. 3, pp. 293-307, 2009. 
[7] A. Bentaib, et al., "Modèle hydrogène pour l'EPS2 : impact des recombineurs sur la risque hydrogène dans l'enceinte de confinement des REP 900 Mwe-Volume I : synthèse des résultats," Note Technique DPEA / SEAC / 2002-35.

[8] E.-A. Reinecke, A. Bentaib, S. Kelm, W. Jahn, N. Meynet, and C. Caroli, "Open issues in the applicability of recombiner experiments and modelling to reactor simulations," Progress in Nuclear Energy, vol. 52, no. 1, pp. 136-147, 2010.

[9] P. Rongier, et al., "Studies of catalytic recombiner performances in H2PAR facility," in Proceedings of the Cooperative Severe Accident Research Program Meeting (CSARP'97), Bethesda, May 1997. 

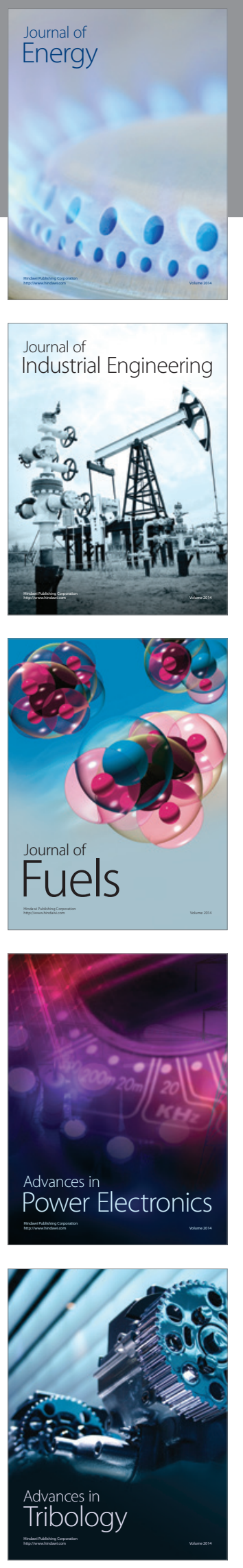
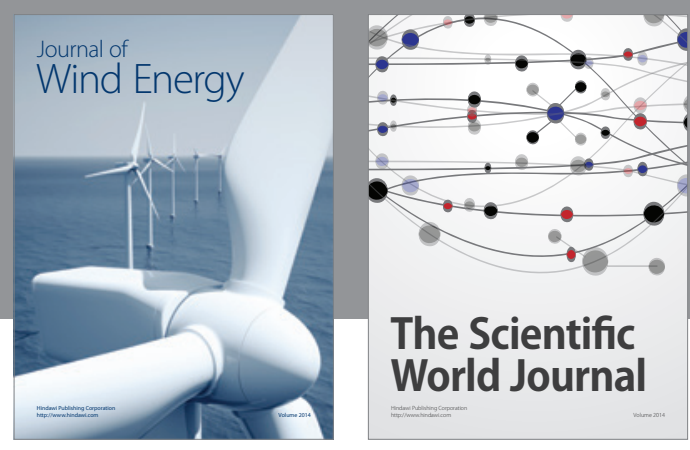

The Scientific World Journal

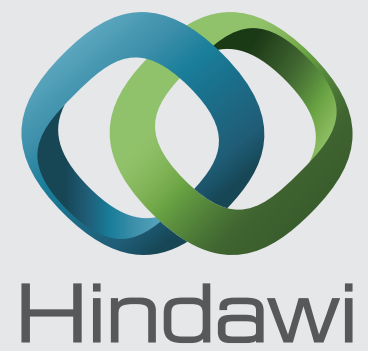

Submit your manuscripts at http://www.hindawi.com
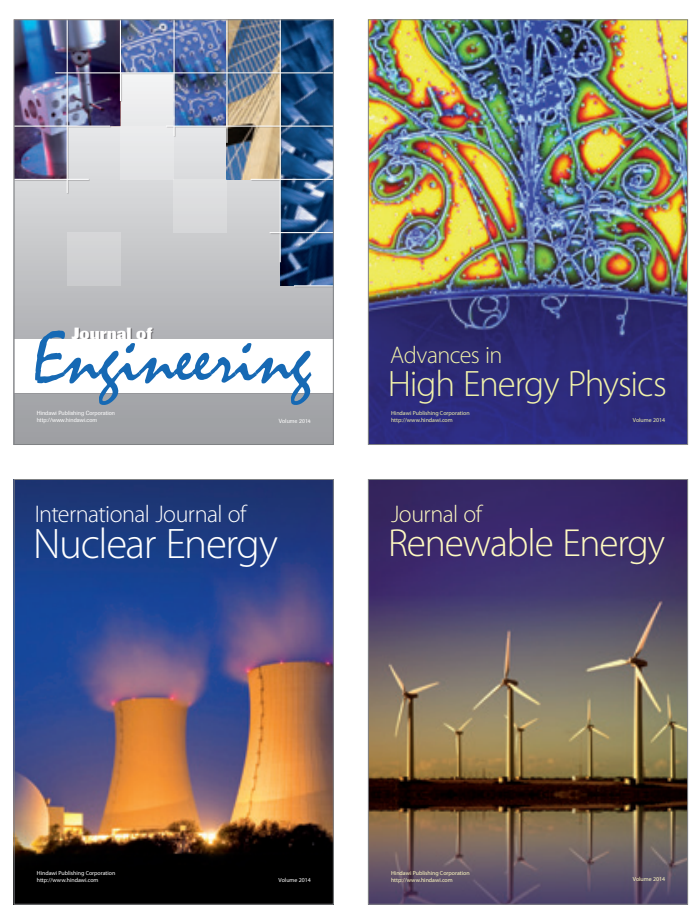

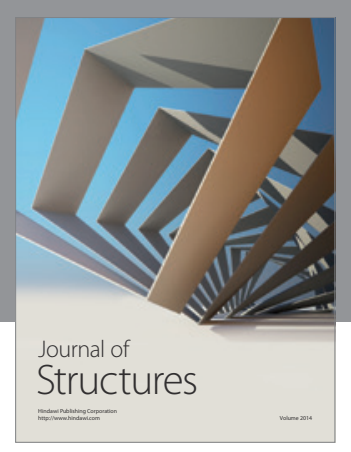

Rotating
Mechinery
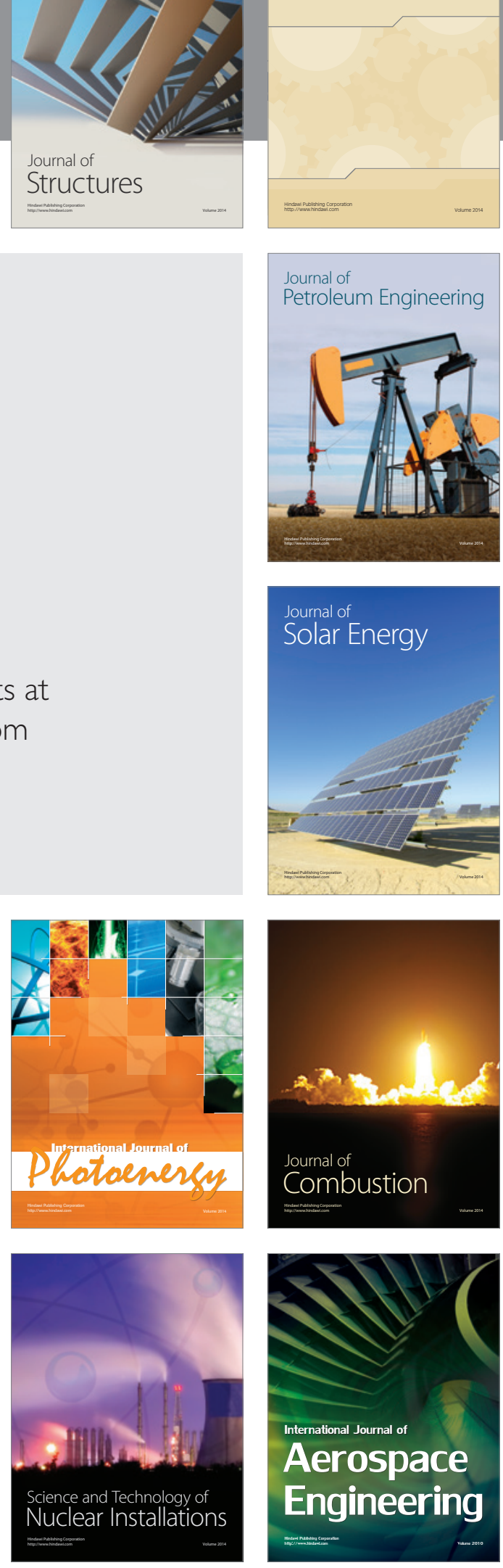